\title{
Numerical optimization of the discrete Mikaelian lens
}

\author{
A. M. Alexandrin, R. O. Ryazantsev, Y. P. Salomatov \\ Institute of Engineering Physics and Radioelectronics \\ Siberian Federal University \\ Krasnoyarsk, Russia \\ e-mail: aalexandrin@sfu-kras.ru
}

\begin{abstract}
The method of the inhomogeneous Mikaelian lens constructing is presented and investigated. The lens is implemented as a discrete medium which is a stack of figured layers of certain geometry, similar to flower with petals. The dependence of aperture efficiency on layer thickness $t$ and the number of the "petals" $N$ was investigated. It was found that discontinuities of the discrete structure representing the lens can exceed the wavelength still retaining high value of aperture efficiency. It was demonstrated that numerical optimization of "petal" profile along with anisotropy accounting can significantly increase the aperture efficiency of the Mikaelian lens.
\end{abstract}

Keywords - inhomogeneous dielectric; lens, lens antenna

\section{INTRODUCTION}

The inhomogeneous dielectric lens is a well-known type of lens antennas with some distinctive features. The most widely used one is the Luneburg lens antenna which allows performing a wide angle undistorted beam steering. Another type of such lens is the Mikaelian lens [1] which is a fragment of self-focusing waveguide. The lens has cylindrical shape with permittivity changing along its radius.

In their recent works authors have demonstrated some applications of the Mikaelian lens [2]. The lens can be used as a superstrate in a sparse antenna array with the period of more than one wavelength. Such a lens can narrow the pattern of a single element thus effectively cancel the sparse array grating lobes. The Mikaelian lens can provide the least thickness of the superstrate layer and has theoretically frequency independent characteristics.

The lens is implemented as a stack of figured dielectric layers. Fig. 1 shows the way the cylindrical lens is split into thin disks which are then divided into sectors with partial filling ("petals"). Experiments show that an array with periodicity of $2 \lambda$ with the superstrate of these lenses has high directivity within $18-26 \mathrm{GHz}$ band with grating lobes lower than $-12 \mathrm{~dB}$.

In the present paper we investigate the dependence of the Mikaelian lens performance on its geometrical parameters.

\section{GEOMETRICAL PARAMETERS OF THE MIKAELIAN LENS}

The lens implemented as shown on Fig. 1 can be characterized by following parameters:

$N$ - the number of "petals" in the single layer. $N=\pi / \varphi_{0}$ (see Fig. 1, d);

$t$ - the thickness of a single layer;
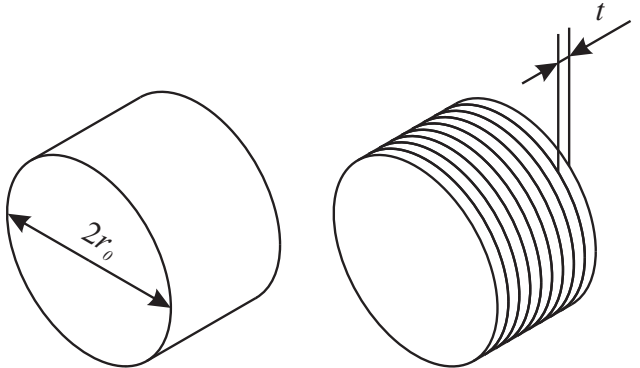

a)

b)

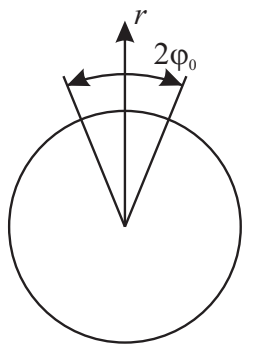

c)

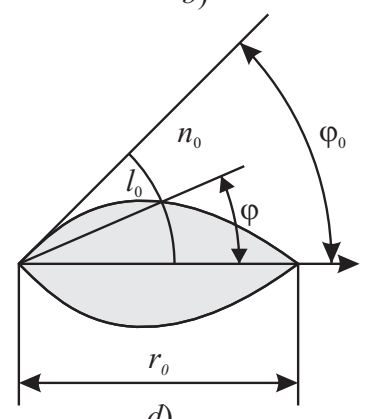

d)

Fig. 1. The implementation of a continuous inhomogeneous Mikaelian lens as a discrete structure. a) - initial cylindrical lens b) - lens split on elementary layers, c) - the layer split on sectors d) - partial filling of the sector by dielectric.

$r_{0}$ - lens radius;

$n_{0}$ - refractive index of a material used for the lens;

The thickness of the lens $L$ is computed as

$$
L=\frac{\pi r_{0}}{2 \operatorname{acosh}\left(\mathrm{n}_{0}\right)}
$$

This implementation of the lens is based on a replacement of a continuous inhomogeneous medium by a discrete homogeneous one. The discrete medium acts like the continuous medium when the discretization is fine enough so that the sizes of discontinuities are much smaller than the wavelength. It means that $N$ must be as high as possible and $t$ must be small enough.

But manufacturing demands are opposite: large $t$ and small $N$, which implies more "rough" lens geometry. Obviously, the choice of $t$ and $N$ is a subject of a compromise between manufacturing demands and demands for finest representation of the inhomogeneous medium. 


\section{NUMERICAL CALCULATIONS OF APERTURE EFFICIENCY}

As a criterion of "quality" of the Mikaelian lens we use its aperture efficiency $e_{a}$. Our task is to investigate the dependence of $e_{a}$ on two geometrical parameters and to formulate the approximate rules for choosing of $t$ and $N$.

Direct numerical calculations of the Mikaelian lens were made in CST MWS. The lens had fixed radius of $20 \mathrm{~mm}$ and was fed by a round waveguide feeder filled with the same dielectric the lens made of $\left(n_{0}=2\right)$. The aperture efficiency was calculated at $30 \mathrm{GHz}$, so the waveguide had the cutoff frequency $20 \mathrm{GHz}$. Calculations were made for radius value of $10,20,30$ and $40 \mathrm{~mm}$.

The elementary layers of the ML are stacked with alternation of their orientation. Fig. 2 shows different cases of such alternation. At the first picture (Fig. 2, a) the layers alternate "by two" so that the adjacent layers are rotated by $\varphi_{0}$. Another case (Fig. 2, b) is the alternation "by three" with rotation of the adjacent layers by the angle $2 / 3 \varphi_{0}$. Cases of $2 / m \cdot \varphi_{0}$ where $m>3$ were not considered due to significant deterioration of the lens characteristics. The third case considered is random rotation of layers (Fig. 2, c).

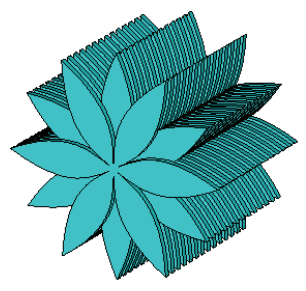

a)

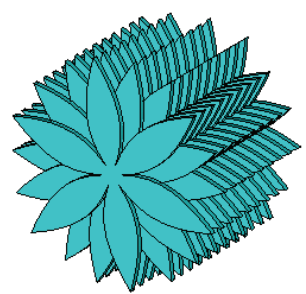

b)

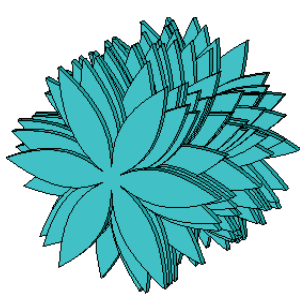

c)

Fig. 2. 3D-models of Mikaelian lens with different layer alternation.

Calculation of the function $e_{\mathrm{a}}(N, t)$ was carried out for tree mentioned methods of layer alternation. The results for $r_{0}=20 \mathrm{~mm}$ can be seen on Fig. 3. The dark red regions correspond to highest level of $e_{\mathrm{a}}$. The results show that increasing of $N$ leads to increase of $e_{a}$ up to a certain limit. After certain value of $N$ further increasing of $N$ almost does not affect $e_{a}$. The same can be said about $t$. Consequently, we can conclude that there are some "threshold" values of $N$ and $t$ that are sufficient for providing maximum level of $e_{a}$. The optimal values of $t$ and $N$ can be chosen as a point within red regions close to their bottom right corner. If we draw a straight line through the origin point and tangent to the region of maximum $e_{a}$, the point of tangency will correspond to optimal $N$ and $t$.
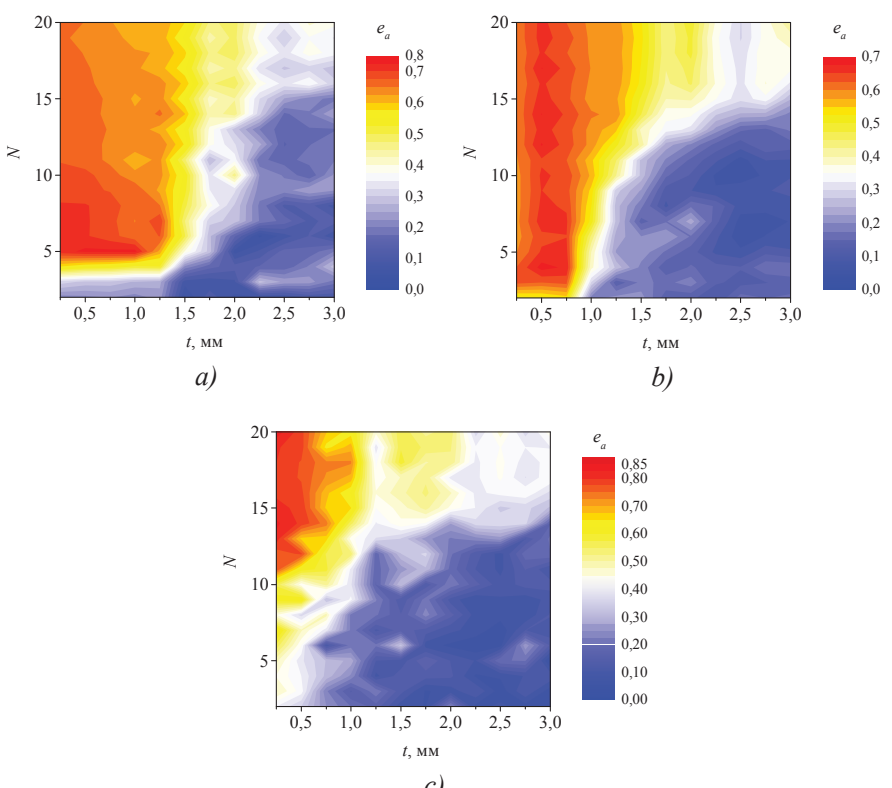

Fig. 3. Aperture efficiency of discrete lens for different layer alternation as a function of layer thickness $t$ and number of "petals" in the layer: alternation of layers by $\varphi_{0}$ (a), by $2 / 3 \varphi_{0}$ (b) and random angle (c)

From Fig. 3 we also can conclude that the best method of lens layers alternating is to rotate them by $\varphi_{0}$ (Fig. 2, a). Thus we achieve maximum of $e_{a}$ with the least values of $N$ and the largest $t$. A comparable level of $e_{a}$ is achievable by random rotation of layers at a cost of significantly higher $N$ and $t$. In the second case (rotating the adjacent layers by $2 / 3 \varphi_{0}$ ) high level of $e_{\mathrm{a}}$ is achieved only for small $r_{0}$ though optimal $N$ is smaller than in other cases.

The results of numerical calculations are summarized in Table I. The value $l_{\max } / \lambda$ corresponds to electric length of arc between the tips of "petals" in the single layer (see Fig. 1):

$$
l_{\max }=2 \phi_{0} r_{0}=2 \pi r_{0} / N \text {. }
$$

TABLE I. PARAMETERS OF LENS AT MAXIMUM APERTURE EFFICIENCY

\begin{tabular}{|c|c|c|c|c|c|}
\hline & $\boldsymbol{r}_{\mathbf{0}} \mathbf{~} \mathbf{m m}$ & $\boldsymbol{t}_{\mathbf{m a x}}, \mathbf{m m}$ & $\boldsymbol{N}_{\mathbf{m i n}}$ & $\boldsymbol{l}_{\mathbf{m a x}} / \boldsymbol{\lambda}$ & $\boldsymbol{e}_{\boldsymbol{a}} \mathbf{m a x}$ \\
\hline \multirow{4}{*}{$\begin{array}{c}\text { Rotation of layers } \\
\text { "by two" }\end{array}$} & 10 & 1,25 & 3 & 2,1 & 0,82 \\
\cline { 2 - 6 } & 20 & 1,25 & 5 & 2,51 & 0,8 \\
\cline { 2 - 6 } & 40 & 0,75 & 8 & 2,4 & 0,7 \\
\hline \multirow{4}{*}{$\begin{array}{c}\text { Rotation of layers } \\
\text { "by three" }\end{array}$} & 20 & 0,75 & 9 & 2,8 & 0,7 \\
\cline { 2 - 6 } & 30 & 0,75 & 3 & 4,2 & 0,7 \\
\cline { 2 - 6 } & 40 & 0,75 & 5 & 5,0 & 0,45 \\
\hline \multirow{3}{*}{$\begin{array}{c}\text { Rotation of layers } \\
\text { by random angle }\end{array}$} & 20 & 0,5 & 12 & 1,0 & 0,8 \\
\cline { 2 - 6 } & 30 & 0,25 & 18 & 1,0 & 0,55 \\
\hline
\end{tabular}




\section{International Siberian Conference on Control and Communications (SIBCON)}

As can be seen from the table, the optimal thickness of the dielectric layers is about $0,2 \lambda_{\text {diel }}$ where $\lambda_{\text {diel }}$ is the wavelength in the dielectric media the lens is made from. The order of the value $l_{\max } / \lambda$ is about $2-2,5$ (here $\lambda$ is the wavelength in the air). From the calculations we can conclude that the discrete representation of inhomogeneous dielectric lens can be made much coarser than it follows from the assumption that all irregularities should be much smaller than wavelength. This significantly simplifies manufacturing of inhomogeneous dielectric lens.

\section{CORRECTION OF ANISOTROPY}

The proposed discrete representation of inhomogeneous medium has one feature: a certain anisotropy. The dependence of the effective dielectric constant on filling coefficient of the sectors, which the single layer split in, is linear for the case of radial polarization (vector $\mathrm{E}$ points along the radius) and slightly nonlinear in the case of orthogonal polarization. Thus the profile of "petals" must be dependent of their orientation with respect to the polarization direction.

This dependence was theoretically calculated in [3], where authors demonstrated possible gain in aperture efficiency for lens with angularly-dependent profile of "petals". In the present work we obtain better results by means of numerical optimization of the "petal" profile in orthogonal planes independently.

The "petal" profile is given by the equation [3]

$$
\varphi(r)=\left(\frac{\varepsilon_{0}}{\cosh ^{2}\left(r \operatorname{acosh}\left(n_{0}\right) / r_{0}\right)}-1\right) \frac{\varphi_{0}}{\varepsilon_{0}-1},
$$

where $\varepsilon_{0}$ is the permittivity of the dielectric. This function can be approximated as a sum of power functions:

$$
\phi(r)=\frac{\phi_{0}}{\sum_{i=1}^{m} c_{i}} \sum_{i=1}^{m} c_{i}\left(1-r^{i}\right)
$$

where $c_{i}$ is an expansion coefficient, $m$ is a number of polynomials in the expansion. It's sufficient to assume $m=3$.

The coefficients $c_{i}$ depend on $N$ and $\varepsilon_{0}$. For $N=8$ and $\varepsilon_{0}=4$ their values are following: $c_{1}=0.41, c_{2}=12.77, c_{3}=-7.58$. These coefficients can be used for aperture efficiency optimization. But if we want to take into account the anisotropy we should independently define the profiles of "petals" in orthogonal planes. So the number of coefficients increases to 6 .

When we independently define the profiles of "petals" in orthogonal planes (e. g. $E$ and $H$ ) the profiles of other "petals" which are not lying in these planes are calculated as weighted average between them with the weights being $\sin ^{2}\left(\varphi_{i}\right)$ for $E$ plane "petal" profile and $\cos ^{2}\left(\varphi_{i}\right)$ for $H$-plane "petal" profile. Here $\varphi_{i}$ is the angle between horizontal plane and the direction of $i$-th petal.
Fig. 4 shows the differences between initial profile calculated from (1) and profile obtained by numerical optimization by CMA ES method. The initial $e_{a}$ of the lens of $20 \mathrm{~mm}$ radius was 0.74 (which corresponds to $20.7 \mathrm{dBi}$ directivity) and after optimization became 0.92 (21.7 dBi). The optimal coefficient values for $E$ and $H$-planes are following:

$$
\begin{array}{lll}
c_{H 1}=-3.64 & c_{H 2}=17.33 & c_{H 3}=-9.13 \\
c_{E 1}=15.29 & c_{E 2}=22.373 & c_{E 3}=-2.06
\end{array}
$$

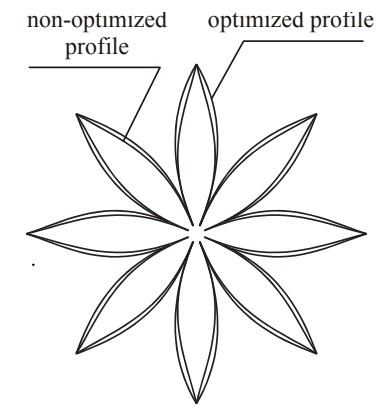

Fig. 4. Initial non-optimized and optimized profiles of lens layer.

\section{CONCLUSION}

In this work we presented two aspects of Mikaelian lens optimization. One of them concerns manufacturing simplification and another - aperture efficiency maximization. Simplicity of manufacturing implies that the geometry of lens components should consists of the least possible number of discrete elements without any loss of aperture efficiency. We demonstrated that the elementary layers forming the lens should have the thickness not more than $0,2 \lambda_{\text {diel }}$, and the number of "petals " in each layer should be such that the arc length between their tips would be about 2 $2,5 \lambda$. It was also demonstrated that the best order of stacking the layers is to alternate the direction of "petals" by angle $\varphi_{0}=\pi / N$ for each two adjacent layers. This provides the least $N$ and the largest $t$ values with the aperture efficiency remaining sufficiently high.

We also demonstrated the way to maximize the aperture efficiency of the lens by approximating the geometry of single "petals" by sum of polynomials with subsequent numerical optimization of the polynomial coefficients. An independent definition of petal profiles in two orthogonal planes allows taking the anisotropy of discrete medium into account. Thus we have reached almost $1 \mathrm{dBi}$ increasing in lens directivity.

\section{REFERENCES}

[1] A. L. Mikaelian, "Self-focusing media with variable index of refracion," Progress in optics, no. XVII, 1980.

[2] A. M. Alexandrin and Y. P. Salomatov, "Wideband array with artificial inhomogeneous dielectric structures," Proceedings of Tomsk State University of Control Systems and Radioelectronics, vol. 1, no. 2(26), 2012.

[3] A. M. Alexandrin, "Implementation of a Radially Inhomogeneous Medium and Construction of the Aperture Antennas on its Basis," 2013 International Siberian Conference on Control and Communications (SIBCON). Proceedings, 12-13 September 2013. 